\title{
PAPER
}

\section{Does desorption affect the length distributions of nanowires?}

To cite this article: V G Dubrovskii et al 2019 Nanotechnology 30475604

View the article online for updates and enhancements.

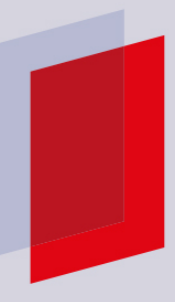

\section{IOP ebooks}

Bringing you innovative digital publishing with leading voices

to create your essential collection of books in STEM research.

Start exploring the collection - download the first chapter of every title for free. 


\title{
Does desorption affect the length distributions of nanowires?
}

\author{
V G Dubrovskii ${ }^{1}$ (1), J Barcus ${ }^{1,2}$, W Kim $^{3}$, J Vukajlovic-Plestina ${ }^{3}$ and \\ A Fontcuberta i Morral ${ }^{3,4}$ (1) \\ ${ }^{1}$ ITMO University, Kronverkskiy pr. 49, 197101 St. Petersburg, Russia \\ ${ }^{2}$ Université Clermont Auvergne, Polytech Clermont-Ferrand, F-63000 Clermont-Ferrand, France \\ ${ }^{3}$ Laboratoire des Matériaux Semiconducteurs, Institue of Materials, Faculty of Engineering, École \\ Polytechnique Fédérale de Lausanne, 1015 Lausanne, Switzerland \\ ${ }^{4}$ Institute of Physics, Faculty of Basic Sciences, École Polytechnique Fédérale de Lausanne, 1015 \\ Lausanne, Switzerland
}

E-mail: dubrovskii@mail.ioffe.ru

Received 9 June 2019, revised 1 August 2019

Accepted for publication 15 August 2019

Published 10 September 2019

\begin{abstract}
State-of-the art models for statistical properties within the nanowire ensembles consider influx of precursors, reflection and surface diffusion of adatoms. These models predict a delay in the nanowire growth start and the evolution toward an asymmetric length distribution. We demonstrate here the effect of desorption of the nanowire material, which has not been considered so far in studies of the nanowire length distributions. We show that at the very beginning of growth the length distribution should be asymmetric due to the slow nucleation of nanowires. At longer times, the length distribution acquires a symmetric Gaussian shape due to the increased weight of desorption. The width of this distribution is larger than Poissonian and increases for higher ratio of desorption over deposition rate. Our model is consistent with the length evolution of organized self-catalyzed GaAs nanowires. We outline that desorption of the nanowire material should be minimized to achieve arrays of highly identical nanowires. These results are relevant for a wide variety of material systems.
\end{abstract}

Keywords: self-catalyzed III-V nanowires, length distribution, group V desorption, growth modeling

(Some figures may appear in colour only in the online journal)

\section{Introduction}

Semiconductor nanowires (NWs), particularly NWs of III-V compounds, are widely considered as fundamental building blocks for nanoscience and nanotechnology [1-3]. Many applications require the highest possible degree of size homogeneity within the ensembles of III-V NWs in terms of both diameter and length. Narrowing the size distributions of these nanoobjects is also interesting from the fundamental viewpoint, with far-reaching generalizations [4]. III-V NWs are usually obtained by the vapor-liquid-solid (VLS) method [5], with either gold [5] or a group III metal (gallium) [6, 7] as the growth catalyst. The ability of gallium droplet to either swell or shrink under low or high V/III flux ratios, respectively [8-12], allows for the fine tuning of NW diameter, including its self-regulation to a very narrow range $[9,10]$.
The accurate prediction of the nanowire length distribution (LD) is much more complex. It is well-known that selfcatalyzed III-V NWs growing at a time and size-independent rate $v=$ const without desorption should have the Poisson LD [13], which is why it is common to compare the measured LDs to the Poisson distribution. There is only one effect leading to a sub-Poissonian narrowing of the NW LD [4], which is associated with a limited number of group $\mathrm{V}$ atoms dissolved in the droplet and called nucleation antibunching $[14,15]$. All other processes, including (i) a nucleation delay of NWs emerging from the substrate [16], (ii) secondary nucleation of group III droplets [16, 17], (iii) surface diffusion of group III atoms [18, 19], and (iv) shadowing effect [20] contribute to broadening of the LDs. Process (iii) does not strongly affect the LDs of self-catalyzed III-V NWs because their axial growth rate is determined by the kinetics of group 
$\mathrm{V}$ species [6, 8-10]. For self-catalyzed III-V NWs grown in organized arrays on patterned $\mathrm{SiO}_{x} / \mathrm{Si}(111)$ substrates with gallium pre-deposition [11, 12, 21], process (ii) is disabled, while process (iv) is unimportant for short enough NWs. Therefore, the main factor for broadening the LDs is the nucleation delay $[4,16]$. Synchronized nucleation of GaAs NWs obtained by different techniques has recently enabled to achieve sub-Poissonian LDs [20, 22]. For long nucleation delays, the LDs acquire a broad asymmetrical shape with a long left tail for NWs which have emerged later $[4,16]$. Without nucleation antibunching, this asymmetry and broadening is described by the single parameter $\alpha=v_{0} / v<1$ [16], which shows how the growth rate of the very first $\mathrm{NW}$ monolayer $(\mathrm{ML})\left(v_{0}\right)$ is delayed with respect to upper MLs (v).

This picture is, however, incomplete, because many experimental LDs, for example, in the case of self-catalyzed GaAs NWs, show a different shape in the initial growth stage $[20,21]$. This shape is more symmetrical than the $\alpha$-distribution given in [16], but much broader than the Poisson LD. Consequently, in this work we study 'desorption' of the nanowire material [23-27] as an additional source for broadening the NW LDs and derive a more general analytic LD. We introduce this desorption by considering a probability that the last monolayer (ML) of a NW disappear; which is a stochastic process symmetric to nucleation of MLs. Desorption of the NW material can manifest through different kinetic processes, such as desorption of arsenic [23, 24, 27] or cadmium [25] atoms from a catalyst droplet, desorption of arsenic adatoms from the NW sidewalls [24], or the reverse diffusion of group III atoms from a catalyst droplet onto the NW sidewalls [26]. The NW grows when the desorption rate is lower than the arrival rate of the NW material, and decays otherwise. In this terminology, desorption of the NW material is equivalent to 'negative' growth considered earlier in [26]. In the particular case of self-catalyzed III-V NWs, the NW MLs can be erased by lowering or even turning off the material fluxes, where arsenic atoms most probably desorb directly from the gallium droplet and the excess gallium atoms may diffuse down to the substrate. These considerations are consistent with the general growth theory, in which a probability of decay (removing the NW MLs in our case) is considered which equalizes the growth rate (adding the NW MLs) at equilibrium [3]. Therefore, the term 'desorption of the NW material' will be used in what follows to describe the VLS systems which are able to dissolve the NW MLs and desorb the excess material through the catalyst droplet.

We show that the shape and width of the new LD is controlled by the two parameters $\alpha$ and $\beta$, describing the nucleation delay and desorption, respectively. The modified LD may change its shape from very asymmetrical at the very beginning of growth to the precisely symmetrical Gaussian, whose width is much broader than the Poissonian and is determined by the desorption rate. With this LD, we are able to fit experimental data on short self-catalyzed GaAs NWs [21], which would be impossible with the $\alpha$-distribution.

\section{Model}

Let us consider self-catalyzed III-V NWs or, more generally, NWs whose axial growth rate is given by the difference between the length-independent deposition rate $v$ (the probability of adding the NW ML per unit time) length-independent desorption rate $v_{\text {des }}$ (the probability of removing the ML per unit time). Introducing the dimensionless variable $\tau=v t$, which equals the deposition rate $v$ multiplied by the growth time $t$, the discrete distribution over the lengths $s$ measured in MLs obeys the system of rate equations (REs)

$$
\frac{d f_{s}(\tau)}{d \tau}=f_{s-1}(\tau)-(1+\beta) f_{s}(\tau)+\beta f_{s+1}(\tau), s \geqslant 2 .
$$

Here, $\beta=v_{\text {des }} / v$ is the desorption fraction, the major factor influencing the shapes of the NW LDs. It will be considered time-independent in what follows, however, the generalization for $\beta=\beta(\tau)$ with saturation at a certain value for large $\tau$ does not significantly change the LD shapes. According to the above discussion, equation (1) means that the population of NWs having the length $s$ increases by adding one ML of semiconductor material to NWs of length $s-1$ and removing $1 \mathrm{ML}$ from NWs of length $s+1$, and decreases when $1 \mathrm{ML}$ is added to or removed from NWs of length $s$. Axial NW growth occurs when $\beta<1, \beta=1$ corresponds to the equilibrium vapor flux which equalizes the desorption flux, while $\beta>1$ yields negative NW growth by evaporation through a catalyst droplet [26].

In the continuum limit $(s \gg 1)$, these discrete REs are reduced to one Fokker-Planck equation of the form

$$
\frac{\partial f(s, \tau)}{\partial \tau}=-(1-\beta) \frac{\partial f(s, \tau)}{\partial s}+\frac{(1+\beta)}{2} \frac{\partial^{2} f(s, \tau)}{\partial s^{2}} .
$$

Following the general method [28, 29], we first obtain Green's function of equation (2) $F(s, \tau)$, which corresponds to the delta-like initial condition at zero time, $F(s, \tau=0)=$ $\delta(s)$. The exact result for Green's function is given by the Gaussian

$$
F(s, \tau)=\frac{1}{\sqrt{2 \pi(1+\beta) \tau}} \exp \left[-\frac{(s-(1-\beta) \tau)^{2}}{(1+\beta) \tau}\right],
$$

with the mean length $\langle s\rangle_{0}=(1-\beta) \tau$ and variance $\sigma_{0}^{2}=(1+\beta) \tau$. Considering the case of positive NW growth $(\beta<1)$, the ratio $\sigma_{0}^{2} /\langle s\rangle_{0}=(1+\beta) /(1-\beta)$ is larger than unity and increases for larger $\beta$. The Poisson case with $\langle s\rangle_{P}=\sigma_{P}^{2}=\tau$ and $\sigma_{P}^{2} /\langle s\rangle_{P}=1$ is recovered only in the absence of desorption $(\beta=0)$. Therefore, desorption of group $\mathrm{V}$ atoms from the droplet decreases the mean length and increases the variance of Green's function with respect to the Poissonian VLS growth [4, 13]. Clearly, Green's function is symmetric around $\langle s\rangle_{0}$. These results become even more obvious if we introduce the new variable $x$ in equation (2) according to $x=(1-\beta) \tau$, for which the regular growth rate equals 1 and the multiplying factor of the second derivative term gives the variance $[(1+\beta) /(1-\beta)] x$. 
The NW LD should now be obtained by convolution of Green's function with the nucleation rate $J(\tau)$ according to [26]

$$
f(s, \tau)=\int_{0}^{\tau} d \tau^{\prime} F\left(s, \tau^{\prime}\right) J\left(\tau-\tau^{\prime}\right) .
$$

Finding the NW nucleation rate in systems with desorption is not as straightforward as in the case $\beta=0$, because the population of droplets $f_{0}(\tau)$ is influenced by removing MLs from NWs of length $s=1$. Numerical tests show, however, that in the interesting case of slow nucleation $(\alpha \ll 1)$ the exponential decay $[4,16]$

$$
J(\tau)=\alpha e^{-\alpha \tau}
$$

provides a good approximation. This feature is explained by the fact that $\alpha \tau$ is the slow variable and hence the corresponding exponential mode is the longest.

Using equations (3) and (5) in (4), after some calculations we get the final result for the NW LD in the form

$$
\begin{aligned}
f(s, \tau)= & \frac{\alpha}{2(1-\beta)} \\
& \times \exp \left\{\frac{\alpha}{1-\beta}[s-(1-\beta) \tau]+\frac{\alpha^{2}(1+\beta)}{2(1-\beta)^{2}} \tau\right\} \\
& \times \operatorname{erfc}\left[\frac{s-(1-\beta) \tau}{\sqrt{2(1+\beta) \tau}}+\frac{\alpha}{2(1-\beta)} \sqrt{2(1+\beta) \tau}\right]
\end{aligned}
$$

with

$$
\operatorname{erfc}(x)=\frac{2}{\sqrt{\pi}} \int_{x}^{\infty} d t e^{-t^{2}}
$$

as the regularized complimentary error function. This LD is controlled by the two parameters $\alpha$ and $\beta$ describing the effects of the nucleation delay and desorption, respectively. It easy to show that the fully formed LD (for which $f(s=0, \tau) \rightarrow 0)$ preserves the normalization

$$
\int_{0}^{\infty} d s f(s, \tau)=1 .
$$

The mean size and variance of the LD are given by

$$
\langle s\rangle=(1-\beta)\left(\tau-\frac{1}{\alpha}\right), \sigma^{2}=(1+\beta) \tau+\frac{(1-\beta)^{2}}{\alpha^{2}} \text {. }
$$

At $\beta=0$, the $\mathrm{LD}$ is reduced to the one-parametric $\alpha$-distribution obtained earlier in [16]. Of course, equation (8) for the mean length makes sense only when $\langle s\rangle$ is positive, which requires that $\tau>1 / \alpha$. This is consistent with the fact that equations (7) and (8) apply only in the large time limit where the NW nucleation is fully completed.

\section{Results and discussion}

According to equation (8), the mean size of the two-parametric LD can be presented as $\langle s\rangle=\langle s\rangle_{0}-(1-\beta) / \alpha$, where $\langle s\rangle_{0}$ is the mean size of Green's function and the second term describes the additional delay of growth due to slow nucleation.

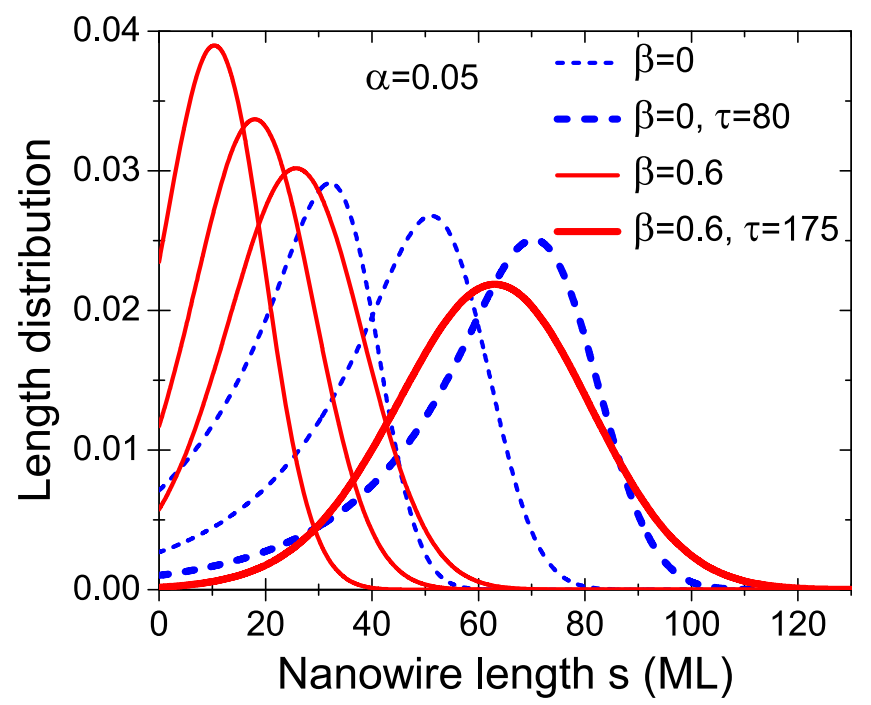

Figure 1. Time evolution of the NW LDs given by equation (6) at $\beta=0$ for $\tau=40,60$ and 80 (dashed lines), and at $\beta=0.6$ for $\tau=$ $40,60,80$ and 186 (solid lines). NWs grow much slower in the presence of desorption $(\beta=0.6)$. Their LDs are asymmetric at the beginning due to slow nucleation (at $\alpha=0.05$ ), and quite similar to the ones in the absence of desorption (at $\beta=0$ ). The mean length of $\sim 65$ MLs is reached at $\tau=80$ for $\beta=0$, and at $\tau=175$ for $\beta=0.6$. The resulting LD shapes, shown by bold lines, are very different - the LD at $\beta=0$ remains asymmetric, with a long tail for shorter NWs, while the LD at $\beta=0.6$ is perfectly symmetric, but broader than the one at $\beta=0$.

The total variance equals $\sigma^{2}=\sigma_{0}^{2}+(1-\beta)^{2} / \alpha^{2}$, where $\sigma_{0}^{2}$ is the variance of the symmetric Green's function, and the second term describes the asymmetry and the additional broadening of the LD due to slow nucleation. For small enough $\alpha$, the $\alpha$-dependent terms in the mean size and variance can be dominant for very short NWs. In a later growth stage, however, the $\langle s\rangle_{0}$ and $\sigma_{0}^{2}$ terms become much greater than the $\alpha$-terms, because both mean size and variance of Green's function are proportional to the growth time $\tau$. This corresponds to a transition from the initially asymmetric LD, influenced by the nucleation delay, to a broad and symmetric Gaussian LD whose width is controlled by desorption of the NW material and can be much larger than in the Poisson case. Therefore, our twoparametric function can describe broad and symmetric NW LDs, while the $\alpha$-distribution remains asymmetric for a much longer time.

These properties are demonstrated in figures 1 and 2 . Figure 1 shows the time evolution of the LDs at $\beta=0$ and $\beta=0.6$ for the same $\alpha=0.05$. It can be seen that the LD shapes are quite similar at the very beginning of growth due to slow nucleation. NWs grow much slower in the presence of desorption, which is why they reach a mean length of $\sim 65$ MLs after $\tau=80$ at $\beta=0$ and only after $\tau=175$ at $\beta=0.6$. The LD shapes at a fixed mean length of 65 MLs become very different-the one at $\beta=0$ remains asymmetric toward a much longer left tail, while the one at $\beta=0.6$ is symmetric around the mean size, but broader than without desorption. Figure 2 shows how the asymmetric LD at $\beta=0$ is transitioned to the symmetric Gaussian with increasing $\beta$ at a fixed mean length of 200 MLs. 


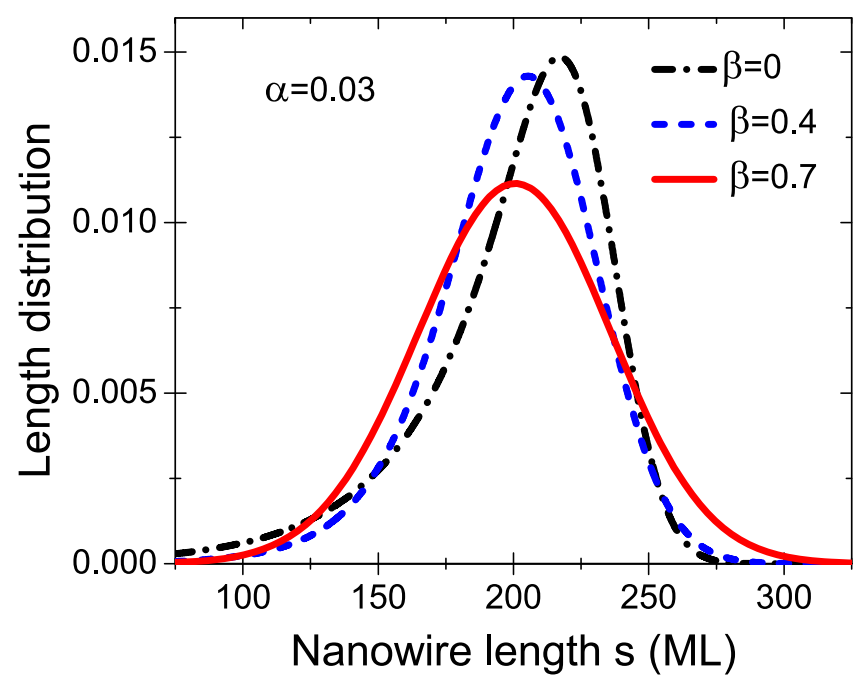

Figure 2. Comparison of the LD shapes at a fixed mean length of 200 MLs for the same $\alpha=0.03$ and different $\beta=0$ (dashed-dotted line), 0.4 (dashed line) and 0.7 (solid line), showing the transition from asymmetric to symmetric LD with increasing the desorption fraction $\beta$.

Figure 3 show a comparison of our model with the experimental data on the LDs of Ga-catalyzed GaAs NWs in the initial stage of their growth. These NWs were grown by molecular beam epitaxy (MBE) in patterned arrays of $45 \mathrm{~nm}$ diameter holes in $\sim 10 \mathrm{~nm}$ thick thermal oxide on $\mathrm{Si}(111)$ substrates. The initial droplets were obtained by gallium predeposition onto the patterned surface. For this series of samples, the gallium atomic flux corresponded to a GaAs planar growth rate of $0.1 \mathrm{~nm} \mathrm{~s}^{-1}$, and the $\mathrm{As}_{4}$ partial pressure was fixed at $2 \times 10^{-6}$ Torr. The substrate temperature during growth was $635^{\circ} \mathrm{C}$. More details can be found in [21] and Methods. At this high temperature, desorption of arsenic atoms is expected to be very significant $[3,23]$. Therefore, we fit the experimental length histograms by our two-parametric LD which accounts for the arsenic desorption.

After 4.5 min of GaAs deposition, the NWs have just started from the holes and their LD is slightly asymmetric. Within our model, this asymmetry corresponds to slow nucleation with $\alpha=0.02$ in equation (6). After $7.5 \mathrm{~min}$ of GaAs deposition, the LDs become symmetric, but much broader than the Poisson LDs. The best fits for the experimental LDs at $7.5 \mathrm{~min}$ and $15 \mathrm{~min}$ are obtained for the same $\alpha=0.02$ and $\beta=0.9 \pm 0.02$. The best fit for $10 \mathrm{~min}$ sample corresponds to $\beta=0.72 \pm 0.02$. These $\beta$ values show that $\sim 72 \%-90 \%$ of deposited arsenic atoms re-evaporate from the gallium droplets. This effect leads to a very significant broadening of the LDs with respect to the Poissonian growth without desorption. According to [21], increasing the $\mathrm{As}_{4}$ pressure from $2 \times 10^{-6}$ Torr to $2.5 \times 10^{-6}$ Torr and further to $3 \times 10^{-6}$ Torr at the same temperature results in a faster axial growth rate and gradual narrowing of the NW LDs. This result is clearly explained by our model, because increasing the influx of arsenic atoms $v$ yields the corresponding decreases of the $\beta=v_{\text {des }} / v$ at $v_{\text {des }} \cong$ const. Quantitative studies of this effect will be presented elsewhere.
Let us now discuss the parameter robustness and the relevance of our two-parametric distribution against other factors which may affect the NW LDs. First of all, there is a large variation in the $\beta$ values obtained from the fits for $4.5 \mathrm{~min} \quad(\beta=0.4)$ and longer $(\beta=0.72-0.9)$ growth times. The $\beta$ variation can be reduced by fitting the histogram at $4.5 \mathrm{~min}$ by equation (6) with $\alpha=0.13$ and $\beta=0.6$, corresponding to the dashed curve in figure $3(\mathrm{~b})$. This curve corresponds to an almost symmetric Gaussian distribution, which is also the case for longer growth times. On the other hand, further decreasing the $\alpha$ parameter with the simultaneous decrease of $\beta$ yields more asymmetric LDs which are not seen in the experiment, as demonstrated by the dashed curve in figure $3(\mathrm{~d})$ at $\alpha=0.002$ and $\beta=0.6$. These examples show that the measured LDs are actually insensitive to the $\alpha$ values, with the LD width being controlled primarily by the $\beta$ parameter. The $\beta$ values range approximately from 0.6 to 0.9 for different samples grown under very similar MBE conditions. All the LDs can be reasonably well fitted by the normal Gaussian distributions [see, for example, figure 3(b)], to which our two-parametric LD is transitioned for longer growth times (see figure 1). In any case, the fits are very sensitive to $\beta$ so that it is impossible to fit the LDs for $4.5 \mathrm{~min}$ and $10 \mathrm{~min}$ samples with $\beta \cong 0.9$ or the LDs for $7.5 \mathrm{~min}$ and $15 \mathrm{~min}$ samples with $\beta=0.6$.

This spread in the $\beta$ values for different samples can be due to several reasons. Local fluctuations in the group $\mathrm{V}$ rates normally enlarge the distribution. However, in our MBE system, the spatial inhomogeneity of the arsenic flux is less than $0.1 \%$ and this effect should be almost negligible. More importantly, our simplified model is restricted to the case of time and radius-independent $v$ and $v_{d e s}$, while the real adsorption-desorption rates may depend on the NW contact angle which changes in the initial stage of growth [21]. Nucleation of MLs within the holes is expected to be different from developed NWs. Although the MBE growth conditions for the considered samples are similar, they were obtained in different growth runs and on different substrates. According to [30], the chemical potential of arsenic atoms in the droplet is strongly affected by the presence of silicon coming from the substrate. The effect is so strong that GaAs NWs cannot grow at all when the silicon concentration is too high. The growth rate of our NWs may also be influenced by silicon atoms, whose concentration may change quite substantially from sample to sample. This depends, for example, on the fraction of clean silicon surface in the hole relative to the oxide surface [30]. Large variation in the widths of both length and diameter distributions observed in [21] can be attributed to the influence of the silicon atoms on the nucleation and growth of self-catalyzed GaAs NWs. All these effects require a separate study which will be presented elsewhere. Given these tolerances and noisy character of the measured length statistics, the variation of $\beta$ from 0.6 and 0.9 is entirely possible and clearly demonstrates the effect of desorption on the NW LDs.

In conclusion, the obtained LD is controlled by the two parameters $\alpha$ and $\beta$ describing the nucleation delay for the very first NW ML and desorption of the NW material (corresponding 

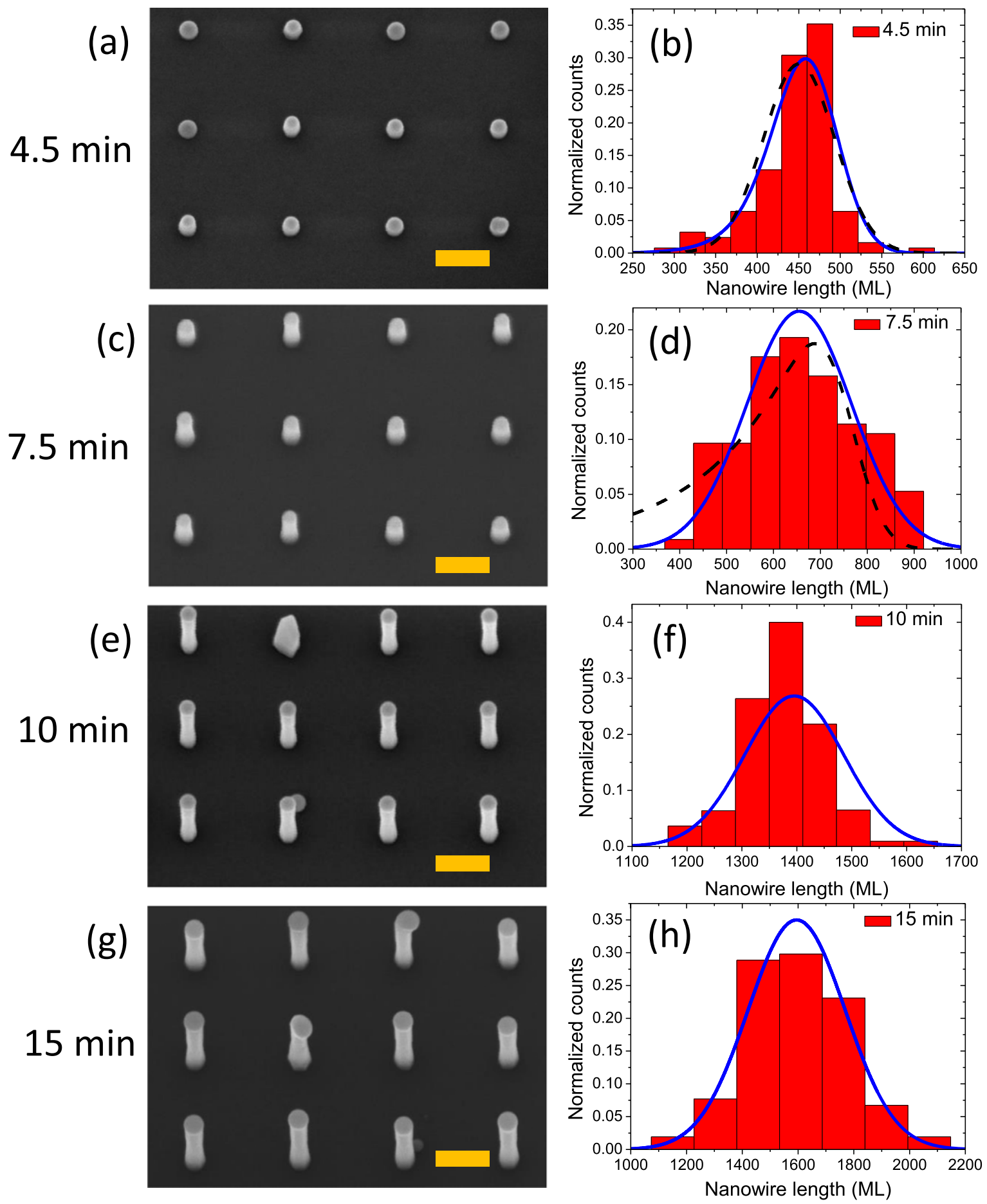

Figure 3. $20^{\circ}$ tilted scanning electron microscopy images of Ga-catalyzed GaAs NWs grown by $\mathrm{MBE}$ in patterned arrays on $\mathrm{SiO} x / \mathrm{Si}(111)$ (left), and their LDs (right) after $4.5 \mathrm{~min}, 7.5 \mathrm{~min}, 10 \mathrm{~min}$, and $15 \mathrm{~min}$ of GaAs deposition, respectively. Scale bars in the images correspond to $200 \mathrm{~nm}$. After the NWs have just emerged from the substrate $(4.5 \mathrm{~min})$, their LD is slightly elongated toward shorter lengths due to difficult nucleation. The best fit to this LD is obtained from equation (6) with $\alpha=0.02$ and $\beta=0.4$. After $7.5,10$ and 15 min of GaAs deposition, the measured length histograms become almost perfectly symmetric, and best fitted by equation (6) with the same $\alpha=0.02$, $\beta=0.9$ for 7.5 and 15 min samples and $\beta=0.7$ for $10 \mathrm{~min}$ sample. The dashed curves show the fits obtained with the modified parameters as discussed in the main text.

to removal of NW MLs), respectively. The effect of desorption becomes more important in the large time limit, and leads to a significant broadening of the symmetric LDs with respect to the Poissonian case. This two-parametric LD is not necessarily restricted to self-catalyzed NWs and can be applied in a more general case of any NWs growing in the VLS regime with desorption of semiconductor material. Clearly, the effect of desorption should be minimized for growing more length-uniform ensembles of NWs, for example, by decreasing the growth temperature or increasing the deposition rate. In our analysis, we 
disregarded nucleation antibunching, in which case the best size homogeneity corresponds to the Poisson LD. According to [27], the effect of antibunching is less in systems with desorption. We now plan to consider an interesting interplay of the nucleation delay, nucleation antibunching and desorption from the viewpoint of the obtained results.

\section{Methods}

The growth substrates were $\langle 111\rangle$ p-doped silicon wafers with a resistivity of $0.1-0.5 \Omega \mathrm{cm}$ and with a $15 \mathrm{~nm}$ thermal oxide (in a Centrotherm furnace at $950{ }^{\circ} \mathrm{C}$ ). The nanoscale pattern was predefined in a ZEP resist with electron-beam lithography. It was transferred on the oxide layer by a $6 \mathrm{~s}$ dry etching using $\mathrm{CHF}_{3} / \mathrm{SF}_{6}$ chemistry followed by $2 \mathrm{~s}$ dip in $7: 1$ buffered hydrofluoric acid solution (BHF). Electron-beam resist was removed by oxygen plasma. In order to ensure an oxide-free surface in the holes, the patterned silicon substrates were shortly dipped in a BHF solution prior to the introduction to the UHV chamber. The final thickness of oxide before loading was $10 \pm 1 \mathrm{~nm}$. After loading into the MBE chamber, the substrates were annealed at $500{ }^{\circ} \mathrm{C}$ for $2 \mathrm{~h}$ in UHV to ensure a pristine surface free of water and organic molecules. The substrate were then transferred to the growth chamber, where prior to growth they were degassed at $770{ }^{\circ} \mathrm{C}$ for $30 \mathrm{~min}$ to further remove any possible surface contaminants.

Measurements of the NW lengths were performed as described in [21]. However, the measured NW lengths given in figure 3 include the droplet heights. This accounts for approximately the same heights of GaAs crystals grown within the holes below the oxide surface. The NW lengths are given in MLs, with a ML height of $0.326 \mathrm{~nm}$ for zincblende $\langle 111\rangle$-oriented GaAs NWs used for the conversion.

\section{Acknowledgments}

VGD gratefully acknowledges the Russian Science Foundation for financial support under the Grant 19-72-30004. JVP, WK and AFiM thank SNF for funding through the NCCR QSIT and H2020 via projects Indeed and Nanoembrace.

\section{ORCID iDs}

V G Dubrovskii (1) https://orcid.org/0000-0003-2088-7158

A Fontcuberta i Morral (i) https://orcid.org/0000-00025070-2196

\section{References}

[1] Yang P, Yan R and Fardy M 2010 Semiconductor nanowires: what's next? Nano Lett. 10 1529-36

[2] Zhang A, Zheng G and Lieber C M 2016 Nanowires: Building Blocks for Nanoscience and Nanotechnology (Berlin: Springer)
[3] Dubrovskii V G 2015 Theory of VLS growth of compound semiconductors Semiconductors and Semimetals ed A Fontcuberta i Morral et al vol 93 (New York: Academic) pp $1-78$

[4] Glas F and Dubrovskii V G 2017 Self-narrowing of size distributions of nanostructures by nucleation antibunching Phys. Rev. Mater. 1036003

[5] Wagner R S and Ellis W C 1964 Vapor-liquid-solid mechanism of single crystal growth Appl. Phys. Lett. 489

[6] Colombo C, Spirkoska D, Frimmer M, Abstreiter G and Fontcuberta i Morral A 2008 Ga-assisted catalyst-free growth mechanism of GaAs nanowires by molecular beam epitaxy Phys. Rev. 77155326

[7] Jabeen F, Grillo V, Rubini S and Martelli F 2008 Selfcatalyzed growth of GaAs nanowires on cleaved Si by molecular beam epitaxy Nanotechnology 19275711

[8] Priante G, Ambrosini S, Dubrovskii V G, Franciosi A and Rubini S 2013 Stopping and resuming at will the growth of GaAs nanowires Cryst. Growth Des. 13 3976-84

[9] Dubrovskii V G, Xu T, Díaz Álvarez A, Plissard S, Caroff P, Glas F and Grandidier B 2015 Self-equilibration of the diameter of Ga-catalyzed GaAs nanowires Nano Lett. 15 $5580-4$

[10] Tersoff J 2015 Stable self-catalyzed growth of III-V nanowires Nano Lett. 15 6609-13

[11] Kim W, Dubrovskii V G, Vukajlovic-Plestina J, Tütüncüoglu G, Francaviglia L, Güniat L, Potts H, Friedl M, Leran J B and Fontcuberta i Morral A 2018 Bi-stability of contact angle and its role in achieving quantum-thin selfassisted GaAs nanowires Nano Lett. 18 49-57

[12] Leshchenko E D, Kuyanov P, LaPierre R R and Dubrovskii V G 2018 Tuning the morphology of selfassisted GaP nanowires Nanotechnology 29225603

[13] Dubrovskii V G 2013 Self-regulated pulsed nucleation in catalyzed nanowire growth Phys. Rev. 87195426

[14] Glas F, Harmand J C and Patriarche G 2010 Nucleation antibunching in catalyst-assisted nanowire growth Phys. Rev. Lett. 104135501

[15] Glas F 2014 Statistics of sub-Poissonian nucleation in a nanophase Phys. Rev. 90125406

[16] Dubrovskii V G, Sibirev N V, Berdnikov Y, Gomes U P, Ercolani D, Zannier V and Sorba L 2016 Length distributions of Au-catalyzed and In-catalyzed InAs nanowires Nanotechnology 27375602

[17] Matteini F, Dubrovskii V G, Rüffer D, Tütüncüoğlu G, Fontana Y and Fontcuberta i Morral A 2015 Tailoring the diameter and density of self-catalyzed GaAs nanowires on silicon Nanotechnology 26105603

[18] Dubrovskii V G, Berdnikov Y, Schmidtbauer Y, Borg M, Storm K, Deppert K and Johansson J 2016 Length distributions of nanowires growing by surface diffusion Cryst. Growth Des. 16 2167-72

[19] Dubrovskii V G 2017 Length distributions of nanowires: effects of surface diffusion versus nucleation delay J. Cryst. Growth 463 139-44

[20] Koivusalo E S, Hakkarainen T V, Guina M and Dubrovskii V G 2017 Sub-Poissonian narrowing of length distributions realized in Ga-catalyzed GaAs nanowires Nano Lett. 17 5350-6

[21] Vukajlovic-Plestina J, Kim W, Dubrovskii V G, Tütüncüoğlu G, Lagier M, Potts H, Friedl M and Fontcuberta i Morral A 2017 Engineering the size distributions of ordered GaAs nanowires on silicon Nano Lett. 17 4101-8

[22] Tauchnitz T, Berdnikov Y, Dubrovskii V G, Schneider H, Helm M and Dimakis E 2018 A simple route to synchronized nucleation of self-catalyzed GaAs nanowires on silicon for sub-Poissonian length distributions Nanotechnology 29504004 
[23] Glas F, Ramdani M R, Patriarche G and Harmand J C 2013 Predictive modeling of self-catalyzed III-V nanowire growth Phys. Rev. 88195304

[24] Ramdani M R, Harmand J C, Glas F, Patriarche G and Travers L 2013 Arsenic pathways in self-catalyzed growth of GaAs nanowires Cryst. Growth Des. 1391

[25] Orrù M, Robin E, Den Hertog M, Moratis K, Genuist Y, André R, Ferrand D, Cibert J and Bellet-Amalric E 2018 Nanowire growth and sublimation: CdTe quantum dots in ZnTe nanowires Phys. Rev. Mater. 2043404

[26] Dubrovskii V G et al 2009 Role of non-linear effects in nanowire growth and crystal phase Phys. Rev. 80205305
[27] Sibirev N V, Nazarenko M V, Zeze D A and Dubrovskii V G 2013 Modeling the nucleation statistics in vapor-liquid-solid nanowires J. Cryst. Growth $\mathbf{4 0 1}$ 51-5

[28] Dubrovskii V G 2009 Fluctuation-induced spreading of size distribution in condensation kinetics J. Chem. Phys. 131 164514

[29] Dubrovskii V G 2019 Analytic form of the size distribution in irreversible growth of nanoparticles Phys. Rev. E 99 012105

[30] Hijazi $\mathrm{H}$ et al 2018 Influence of silicon on the nucleation rate of GaAs nanowires on silicon substrates J. Phys. Chem. 122 19230 